\title{
Current Evidence on Superior Capsular Reconstruction
}

\author{
Luísa Pinto ${ }^{1 *}$ and João Torres ${ }^{1,2}$ \\ ${ }^{1}$ Faculty of Medicine, University of Porto, Porto, Portugal \\ ${ }^{2}$ Department of Orthopedics, Hospital de S João, Porto, Portugal
}

\begin{abstract}
Massive irreparable rotator cuff tears remain one of the most challenging pathologies in shoulder surgery to treat, especially in the younger active patients. Although several treatment options exist, none provides superior long-term results. Over the last few years, a promising technique has emerged-the Superior Capsular Reconstruction (SCR). This technique reinforces the superior capsule to keep the humeral head centered within the glenoid, optimizing the force couples necessary for dynamic shoulder function and preventing the proximal migration of the humeral head and, consequently, subacromial impingement. Since SCR is technically challenging and difficult to reproduce consistently, there is a continuous improvement of arthroscopic techniques. So far, SCR has been shown to be effective for pain relief and restoration of active shoulder motion, but mid- and long-term outcomes have not yet been reported.
\end{abstract}

\section{Keywords}

Shoulder, Massive rotator cuff repair, Superior Capsular Reconstruction, Graft, Surgical technique

\section{Abbreviations}

AMC: Anterior Margin Convergence; ASE: American Shoulder and Elbow Surgeons Score; PMC: Posterior Margin Convergence; RSA: Reverse Shoulder Arthroplasty; SCR-Superior Capsular Reconstruction; VAS: Visual Analogue Scale

\section{Methods}

The search was performed between October 2017 and January 2018 using the following query on Pubmed's database: "Superior Capsular Reconstruction", "Superior Capsule Reconstruction", "Massive Irreparable Rotator cuff tear", "Treatment", "Graft", "Patch", "Dermal allograft", "Surgical technique". The restrictions applied were: English Language and Publication date in the last 10 years. Clinical Trials, reviews and systematics reviews were included. From the primary selection, based on the title, 52 articles were chosen. However, from these 52, only 31 abstracts were considered relevant and used as a reference in this review.

Bibliographical references in the selected articles were reviewed and 1 of them was added to the repertoire of this work. Additionally, 1 biomechanical study from Arthrex was used. Thus, this monograph was based on 33 references.

\section{Introduction}

Rotator cuff tears are one of the most common shoulder injuries. The prevalence increases with age as shown by imaging studies reporting rotator cuff tears in up to $30 \%$ of individuals aged 60 years or more and up to $50 \%$ in people aged 80 years or more [1]. Various classifications of rotator cuff tears have been proposed. These tears are usually classified by their anterior-posterior size: $<1 \mathrm{~cm}, 1-3 \mathrm{~cm}, 3-5$ $\mathrm{cm}$, and $>5 \mathrm{~cm}$, respectively classified as small, medium, large and massive [1,2]. Many authors define tears as massive if there is a detachment of at least 2 complete tendons [2-4].

More than $30 \%$ of rotator cuff tears are massive/irreparable tears with a retear rate after the first surgery that ranges from 20 to $90 \%$ [5,6]. A rotator cuff tear is considered irreparable when the torn tendon cannot reach the original footprint on the humeros, due to tendon retraction, muscle atrophy and fatty infiltration [1]. Therefore, surgical success depends on these three variables, tendon quality, patient's age, tear size and the repair technique that is chosen $[3,7]$. The effects of these variables are often additive, which makes the likelihood of healing more difficult to predict [8]. Though the final decision on reparability of the rotator cuff is made intraoperatively, some signs and radiologic findings may suggest irreparability before the surgery [4]. Clinical signs, which suggest that a repair is likely to fail, include static anterosuperior subluxation and associated pseudoparalysis on ante-

*Corresponding author: Luísa Pinto, Faculty of Medicine, University of Porto, Rua Padre Arnaldo Rebelo $n^{\circ} 32$, Vermoim-Maia, Porto, 4470-249, Portugal, Tel: 00351915749697

Accepted: June 12, 2019

Published online: June 14, 2019

Citation: Pinto L, Torres J (2019) Current Evidence on Superior Capsular Reconstruction. Arch Sports Med 3(1):149-155 
rior elevation, dynamic anterosuperior subluxation of the humerus upon resisted abduction [4], a lag sign on external rotation and a positive Hornblowers sign [3]. An acromiohumeral interval of less than $7 \mathrm{~mm}$ on a standard anterior posterior shoulder radiograph, is highly suggestive that a repair may be unsuccessful [9]. Increased age is also associated with poor tendon-to-bone healing, probably because of poor tendon and bone quality, poor vascularity of the tendon and decreased capacity to produce healing growth factors [8].

Although the presence of a preoperative tangent sign and a Goutallier 3-4 fatty infiltration of the supraspinatus is often associated with irreparability, most of the tears with these preoperative characteristics (70\% and $57 \%$, respectively) have been described as completely reparable intraoperatively [10]. Thus, surgeons should be careful about presuming the ability to correctly predict the intraoperative irreparability of rotator cuff tears.

The lack of clear evidence on how to manage irreparable massive cuff tears especially in patients younger than 65 years has led to a lot of suggested treatments, including debridement and subacromial decompression, partial repair, reverse shoulder arthroplasty and various tendon transfers (the most common being the use of latissimus dorsi) [2,3,5,1113]. However, all alternatives to the complete repair of the rotator cuff have showed clinically inferior results and higher complication rates [14].

Patients with irreparable rotator cuff tears also have a defect of the superior capsule, which is located on the inferior surface of the supraspinatus and infraspinatus tendons $[7,15]$ and attached to a substantial area of the greater tuberosity (30\% to $61 \%$ ) [14]. On one hand, the rotator cuff, deltoid and biceps muscles are responsible for keeping the force couples and the humeral head centered within the glenoid during movement (dynamic stabilizers of the shoulder) [16]. On the other hand, the superior shoulder capsule is the main static stabilizer of the glenohumeral joint [14].

The natural history of massive rotator cuff tears consists in the continuous superior migration of the humeral head, due to the instability of the glenohumeral joint, resulting in pain, subacromial impingement, muscle weakness, loss of motion and functional limitations, that can evolve to pseudoparalysis $[7,14,17]$. Biomechanically, it has been shown that superior capsular defects increased glenohumeral translation in all directions, particularly with superior translation at $5^{\circ}$ and $30^{\circ}$ of abduction [11].

Mihata, et al. [7,14] in 2007 described the Superior Capsular Reconstruction (SCR) for the treatment of patients without arthropathy and with irreparable tears. The SCR technique reinforces the superior capsule to keep the humeral head reduced in the glenoid by connecting the glenoid to the greater tuberosity. This surgical technique provides the potential to rebalance the force couples necessary for dynamic shoulder function [15], improving the shoulder function, specially deltoid function [4], and it has also outstanding outcomes in pain [17].
Currently, orthopaedic surgeons have rapidly embraced SCR, as evidenced by more than 10,000 cases worldwide being reported by a single implant vendor for this procedure since 2014 to this day [10].

\section{Surgical Technique}

\section{General}

Mihata, et al. [18], in a biomechanical study, reported that SCR can increase superior stability of the shoulder joint when the graft is attached at either $10^{\circ}$ or $30^{\circ}$ of glenohumeral abduction. Furthermore, a graft attached at a higher degree of shoulder abduction is at increased risk of tearing after SCR because of the high level of tension when the shoulder is adducted. For this reason, the same study considered a glenohumeral abduction of $10^{\circ}$ and $30^{\circ}$ the best arm position [18]. It is also recommended placing the arm in a neutral rotation and neutral flexion, because excess of rotation or flexion-extension changes the distance between the glenoid and the greater tuberosity asymmetrically, from anterior to posterior [17]. With the arm in neutral position, distances between anchors are correctly measured [17].

Surgery is performed with general anesthesia and with an additional interscalene nerve catheter to help with analgesia immediately postoperatively and during the initial rehabilitation process [11].

\section{Technique}

A posterior portal is established and a standard $30^{\circ}$ arthroscope is introduced. A standard diagnostic arthroscopy is performed and any pathology is treated properly [15].

In many tears that are not fully reparable, the subscapular muscle is also involved. However, this muscle is almost always repairable. Thus, subscapular is repaired before approaching the remaining rotator cuff, along with a coracoplasty procedure and biceps tenodesis or tenotomy when indicated [19].

Before the placement of the anchors for the sutures is initiated, the superior glenoid and greater tuberosity need to be prepared to bleeding bone to enhance biologic graftto-bone healing. The superior labrum is left intact to optimize superior stability of the humeral head [20]. After 4 anchors have been placed (typically an anterosuperior and a posterosuperior glenoid anchor and 2 most medial humeral anchors), the dimensions between them are measured to determine the size of the graft. The final graft size should have $5 \mathrm{~mm}$ of extra tissue on 3 sides (medial, anterior, and posterior) in order to prevent the suture from cutting through hand and $10 \mathrm{~mm}$ extra laterally to cover the greater tuberosity $[10,19]$. The graft is then introduced into the shoulder through the anterolateral portal by a cannula, using a double-pulley technique. This is performed by tying 1 limb from each anchor to each other with a static surgeon's knot over a rigid instrument and then advancing down into the shoulder by pulling on the 2 untied suture ends (Figure 1). Once the graft is in its desired position medially, the 2 untied suture ends are tied arthroscopically over the graft, securing its medial attachment [20]. On the humeral side, two 


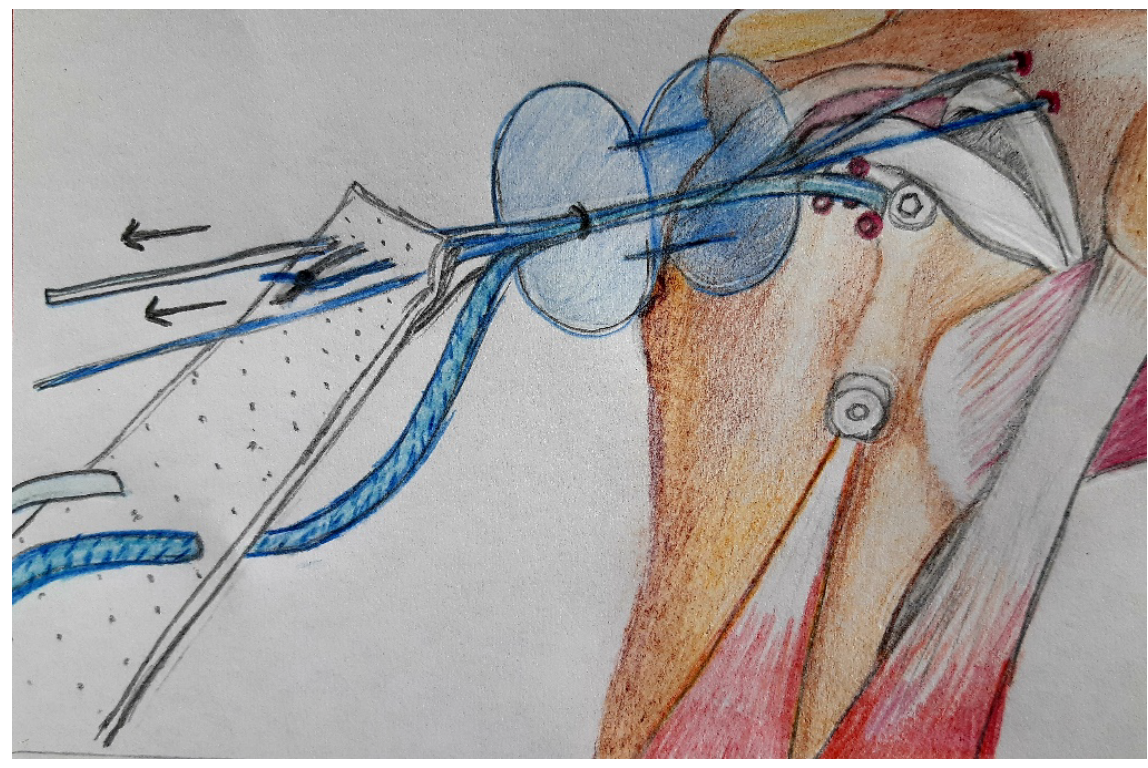

Figure 1: The graft is pulled into the shoulder using a double-pulley technique.

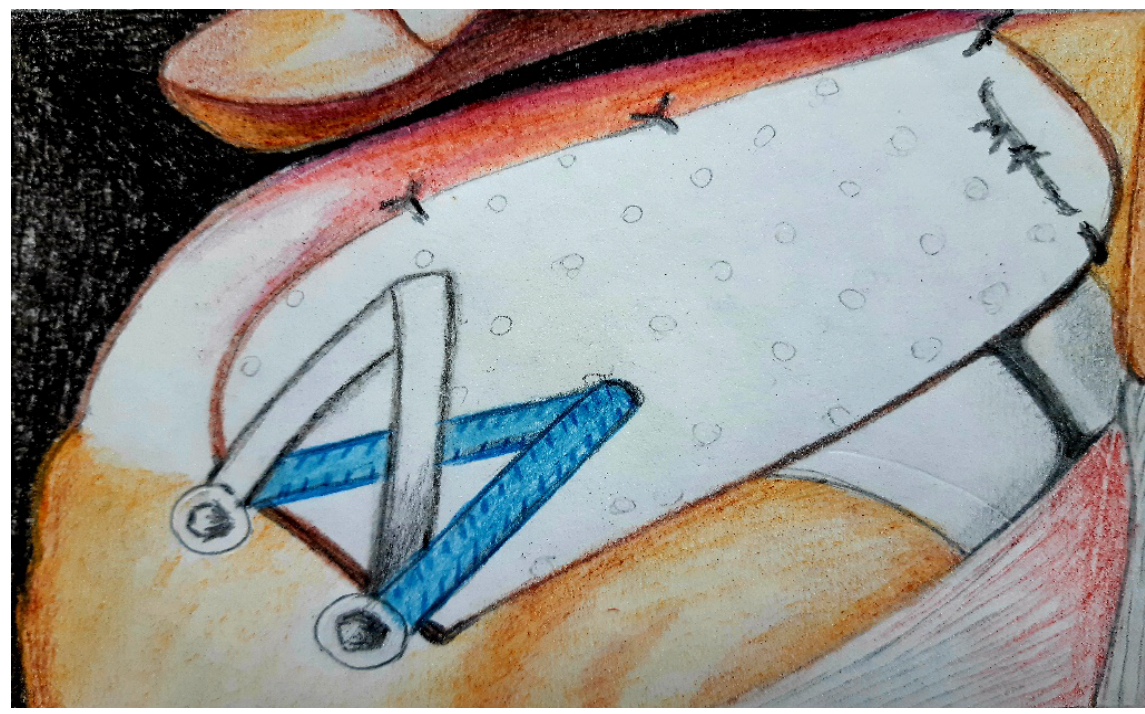

Figure 2: Humeral fixation using a suture bridge technique and posterior margin convergence.

anchors were inserted for medial-row fixation at the articular cartilage margin. Two lateral anchors are then inserted both posteriorly and anteriorly along the lateral rotator cuff footprint on the greater tuberosity for lateral-row fixation. Finally, the lateral part of the graft is compressed down onto the footprint using a suture bridge technique (Figure 2) [15].

In the end, the graft must be sutured posteriorly to the infraspinatus. This is an important and not an optional step, since it is essential for recreating stability (Figure 2) [16].

\section{Zip-line shuttle technique}

When the graft needs to be bigger than $35 \mathrm{~mm}$ in the anteroposterior dimension, it can be so big that it cannot be passed all the way through the cannula and it also can create forces that are strong enough to dislodge the glenoid anchors from the bone. The Zip line technique can overcome these 2 problems. It consists in placing a third glenoid anchor between the anterior and posterior glenoid anchors and push the graft along 2 zip-lines (the 4 sutures from the anterior glenoid anchor; and the 4 sutures from the posterior glenoid anchor) while the 2 sutures from the middle glenoid anchor are used to pull the graft into the shoulder. Tension in these middle-anchor sutures is reduced by having their direction of pull through the modified Neviaser portal instead of the lateral portal. One limb of each suture from the middle glenoid anchor is removed by the lateral portal and a mulberry knot is tied near the end of both of these sutures so that the corresponding free ends of these sutures can be tensioned to help in pulling the graft into the shoulder [19]. Then, a calibrated pusher is used to alternately push the graft down the tensioned anterior and posterior zip-lines [10]. At the same time, the sutures from the middle glenoid anchor are tensioned in order to pull the graft into place over 
the superior glenoid [19].

\section{New evidences}

Mihata, et al. [7,14] have showed that anterior margin convergence (AMC) was not necessary. However, prospective evaluation data began showing improved outcomes with AMC [17]. Graft tension is essential to the success of SCR and it depends on the exact measurement of the distances between the anchors to punch holes in the graft, arm position to set the relationship between the anchor distances, AMC and posterior margin convergence (PMC). When the graft is appropriately secured in the anteroposterior direction through $A M C$ and $P M C$, elongation in the medial-lateral direction will be limited, reducing the elasticity of the graft, improving general stability, and ultimately producing better clinical outcomes [17].

As this technique is developing, a variety of techniques have been suggested for glenoid-side fixation [21]. The medial sutures may be secured with a variety of methods, such as simple knots or mattress sutures [16]. However, it is recommended for glenoid fixation adding an anchor and double-row fixation when it is possible, on the grounds that 3 anchors and a double-row construct are biomechanically stronger [17].

A recent case report was the first to describe the incorporation of the remnant of the superior rotator cuff into the graft, which may be beneficial for the graft incorporation, as it can improve the biologic environment. The authors recommend that sutures should be placed within the mid portion of the graft for superior repair of a residual tendon when it is present. Further research is still needed [22].

\section{Graft}

The initial success of the SCR started a renewed interest in patches for rotator cuff surgery [23].

There is continuing research about the most efficient and reliable material to reconstruct the capsule. Xenograft, allograft and synthetic materials have been tried with the objective of searching for better results and a better healing process $[5,10,13]$. The matrix should be biocompatible in order not to be rejected and it should be properly processed to avoid the risk of disease transmission or immune response [5].

Biological scaffolds are protein-based extracellular matrices that are usually derived from human or animal connective tissues. Advantages of biological scaffolds are a 3D surface proteic microstructure and natural porosity, which provide larger space for host cell attachment, proliferation, migration and assists gas and metabolite diffusion [24]. These properties allow a quicker interaction between biological scaffolds and host tissue. In this way, the formation of new tissue is faster than in synthetic scaffolds [24].

Not all acellular matrices are identical since there are different processing and different natural characteristics of the matrix. Some are made from porcine small intestine submucosa, porcine bladder matrix, bovine dermis and porcine dermis (xenografts), which may not be strong enough to support the SCR [14]. Xenografts have been used to increase the biologic healing in reparable rotator cuff tears, acting as a collagen scaffold [20]. However, there is some potential for an inflammatory response and subsequent failure to heal $[6,20]$.

In biomechanical studies, dermal-based grafts demonstrated higher loads to failure when compared with xenografts and submucosal-based grafts [25].

The SCR technique was originally described using a fascia lata autograft-folded over to produce a thick superior capsule imitation. However, studies have recently shown that using an acellular dermal allograft may be a better choice, because it demonstrated maximum loads to failure 2.5 times greater than the fascia lata autograft [12]. Furthermore, dermal allograft in SCR withstands applied forces and repeated deformations and has excellent clinical outcomes [17]. Mihata also found dermal allograft had a clear advantage in providing a better range of motion, whereas fascia lata autograft resulted in a stiffer construct [22].

The $3 \mathrm{~mm}$ thick acellular dermal allograft is prepared by removing the epidermis and all cellular components. This results in a graft composed of several types of collagen (predominantly type I), chondroitin sulfate, elastin, proteoglycans and fibroblast growth factor. The final graft is characterized by an intact matrix and a basement membrane rich in vascular channel retention [25]. This process reduces the inflammatory response and allows revascularization and cellular repopulation. This matrix is a biologic scaffold, permitting the migration of the adjacent bone marrow's pluripotent cells to form a "neotendon" [13]. This allograft plays a relevant role in rotator cuff healing at the cellular level [2].

The reliable performance of acellular dermal allograft, in terms of elongation and stiffness properties, makes it a practical replacement for autologous fascia lata [21]. Given the excellent mechanical properties of the acelullar dermal allograft and the ability to develop "cuff-like" tissue, the use of the fascia lata has been replaced by many surgeons, thus reducing surgery time and avoiding any donor-site morbidity $[11,26]$.

On the other hand, some studies have shown synthetic grafts to be mechanically stronger than biologic grafts [6]. As they are made from chemical compounds, it is easier to control the chemical and the physical properties leading to quality consistency $[6,24]$. However, biocompatibility of synthetic scaffolds is poor, as they can never be absorbed or integrated into host tissue [24]. High incidences of infection, instability, synovitis, osteolysis, and osteoarthritis have been reported with the use of such materials [27].

\section{Postoperative care}

Postoperative pain control is assisted by an interscalene block or interscalene nerve catheter $[11,28]$.

An early period of immobilization is generally recommended since it has been reported in animals with early immobilization a decreased repair tension, better rotator cuff 
blood supply, improved tissue quality and improved viscoelastic properties [8]. It is recommended an abduction pillow for 4 weeks after the reconstruction [14].

Firstly, the rehabilitation should focus on limited and protected passive range of motion, with an abduction sling. Active and active-assisted movement should begin at 6 weeks postoperatively. Muscle strengthening is recommended after 8 weeks $[11,28]$.

High-demand activities such as golfing, and weight lifting are restricted for 1 year postoperatively. For many patients, SCR is their last opportunity for joint preservation. Thus, Buckhart, et al. [10] prioritize healing of the reconstruction over achieving early range of motion. The main goals of rehabilitation are to improve motion, to prevent scarring, and to improve glenohumeral and scapulothoracic biomechanics [28].

\section{Discussion}

Massive and irreparable rotator cuff tears are not unusual and consist of a difficult problem for orthopedic surgeons $[5,6]$. Despite ample investigation, there is still not a clear treatment option, for this kind of tear, especially in the younger active patients [5].

The most common nonsurgical option for patients with massive tears and without evidence of pseudoparalysis, indicating a well-compensated force couple, consists in subacromial corticosteroid injections or physical therapy to enforce the intact portion of the rotator cuff, deltoid muscle and the periscapular musculature. Although some studies have shown promising results in these patients, other studies have shown poor results of conservative management of massive tears [4]. In a small cohort, Zingg, et al. have documented a significant progression of glenohumeral arthritis, as well as a significant progression in tear size and a decrease of the acromiohumeral interval, despite maintenance of shoulder function and mild pain symptoms. Conservative treatment may lead to a very satisfactory clinical situation in low-demand patients or patients that want to avoid surgery, but it also leads to an inevitable joint degeneration [20].

Partial repair is another option, but the risk of a recurrent tear can be as high as 52\% [15]. Furthermore, a high structural failure rate was shown when partial repairs were imaged at 2 years after the procedure, as well as a modest functional improvement [29]. Burkhart, et al. [30] have found revision and partial rotator cuff repair without SCR to be less reliable in reversing pseudoparalysis than complete repair, primary repair or partial repair in conjunction with SCR. However, it remains a good option for elderly patients wishing pain relief who have preserved overhead function of the shoulder. Many of these patients have comorbidities or poor bone stock, which would make adding SCR an imprudent choice with limited marginal benefit for the patient [10].

Some surgeons opt for Reverse Shoulder Arthroplasty (RSA) in patients without glenohumeral arthritis and with massive tears $[3,25,31]$. However, due to the post-operative restrictions of this procedure, younger patients cannot maintain their quality of life. Furthermore, this is a high risk procedure and leaves no further option if it fails, not to mention the fact that life expectancy of younger patients exceeds the wearing characteristics of the prosthesis [25,31].

This procedure has some known complications, including infection, failure, fracture and neurovascular problems [32]. More precisely, a $38 \%$ complication rate has been reported after RSA among patients less than 65-years-old [21]. Burkhart, et al. concluded that a joint preserving solution might be desired for younger populations since RSA may disrupt the normal anatomy and limit future treatments [19]. Compared with RSA, SCR has fewer risks and fewer complications and does not limit further surgical options [17]. However, RSA has a role as a primary procedure in massive rotator cuff tears without glenohumeral arthritis in some specific cases with a combination of patient and disease factors, such as: Older age (> 60 years), sedentary lifestyle, multiple failed cuff repairs, chronic pseudoparalysis (> 6 months), recurrent shoulder instability or frank anterosuperior escape (subcutaneous) and extensive fatty infiltration of multiple cuff muscles on magnetic resonance imaging. When several of these are present, especially with chronic pseudoparalysis, reverse shoulder replacement is a reliable option for pain relief and restoration of overhead function [10].

Another alternative in younger patients without arthritis is a tendon transfer. Although it has presented promising long term results ( $>10$ years) [33] the overall outcome is still variable with unpredictable results [3]. Debridement with or without biceps tenotomy is a viable option in the elderly and low demand patient, but it was shown that it does not slow the progression of osteoarthritis. Furthermore, it can decrease the strength of arm elevation [4].

Patch graft surgery to the torn tendon has been suggested for the irreparable tears. However, a high rate of patch graft retears has been reported, though various materials have been used including porcine small intestinal submucosa, porcine dermal collagen, and allografts [23]. As shown in the biomechanical study of Mihata, et al. [7], the inter position patch grafting to the torn tendon only partially restored stability. With this procedure, the humeral head still moves superiorly and in this way the patch graft could be abraded and torn by subacromial impingement. On the other hand, attaching the allograft medially to the superior glenoid completely restores superior translation to that of the intact rotator cuff condition [7]. Furthermore, SCR may be a better graft construct because the medial fixation is not to typically poor-quality tendon [23].

Audenaert, et al. reported that the acromio-humeral distance (AHD) did not change significantly after conventional patch graft surgery - AHD, 6.2 to $11.3 \mathrm{~mm}$ preoperatively and 6.7 to $12.8 \mathrm{~mm}$ postoperatively [14]. However, Mihata, et al. [14] reported a significantly increase in AHD, by $4.1+-1.7$ $\mathrm{mm}$, after SCR.

Early published clinical results, and anatomical and biomechanical basic science studies support SCR as a viable surgical option for many patients who previously had few good choices [10].

Most surgical treatments relieve shoulder pain, but pa- 
tients find it difficult to recover muscle strength in elevation and external rotation even after alternative types of surgery, including latissimus dorsi tendon transfer and partial repair [14]. However, the original series by Mihata, et al. [14] with a fascia lata autograft showed good clinical outcomes at a minimum 2-year follow-up in 24 shoulders with irreparable large and massive cuff tears that underwent SCR. The improvements in clinical outcome scores and range of motion were dramatic (mean American Shoulder and Elbow Surgeons score (ASE): 24 preoperative to 93 postoperative, $\mathrm{P}<$ 0.00001 ; active elevation: $84^{\circ}$ preoperative to $157^{\circ}$ postoperative, $\mathrm{P}<0.001$ ), particularly for the $83 \%$ of patients with intact reconstructions and no progression of muscle atrophy on postoperative magnetic resonance imaging.

In another prospective clinical study in 100 patients, ninety-two patients (92\%) had neither graft tear nor re-tear of the repaired rotator cuff tendon during the follow-up period of 5 to 8 years. All patients (26\%) that played sports before their injuries returned fully to their previous sports and pseudoparalysis was reversed in almost all subjects [16].

Hirahara, et al. [17], in a recent study, also have followed patients that underwent SCR, starting before the surgery until 2 years after. The mean ASE score improved significantly $(P<0.00002)$, from 43.54 to 86.46 , and mean VAS pain score decreased significantly $(P<0.00002)$, from 6.25 to 0.38 [17]. The results of this study reflect the clinical outcomes reported by Mihata, et al. [4] and confirm that SCR improves functional outcomes [17].

This surgery has a lot of advantages, such as a very low infection rate, reversal of pseudoparalysis, lower cost than RSA and it does not preclude future RSA if it is necessary [26].

However, SCR is technically challenging, difficult to reproduce consistently and time consuming [29]. Some disadvantages are the difficulties in introducing and securing the matrix in the correct orientation, as well as the difficulty in preventing the patch from folding. Suture management could also be difficult because the space becomes tight and sutures can become intertwisted with each other [29].

\section{Conclusion}

Early clinical and biomechanical research has shown that SCR can be a beneficial treatment option for irreparable rotator cuff tears to restore glenohumeral stability, especially in younger patients $[7,14]$. However, mid-and long-term outcomes have not yet been reported [15].

\section{References}

1. Lewington MR, Ferguson DP, Smith TD, et al. (2017) Graft utilization in the bridging reconstruction of irreparable rotator cuff tears: A systematic review. Am J Sports Med 45: 3149-3157.

2. Gupta AK, Hug K, Berkoff DJ, et al. (2012) Dermal tissue allograft for the repair of massive irreparable rotator cuff tears. Am J sports Med 40: 141-147.

3. Anley CM, Chan SK, Snow M (2014) Arthroscopic treatment options for irreparable rotator cuff tears of the shoulder. World J Orthop 5: 557-565.

4. Gerber C, Wirth SH, Farshad M (2011) Treatment options for massive rotator cuff tears. J Shoulder Elbow Surg 20: S20-S29.

5. Varvitsiotis D, Papaspiliopoulos A, Antipa E, et al. (2015) Results of reconstruction of massive irreparable rotator cuff tears using a fascia lata allograft. Indian J Orthop 49: 304-311.

6. Gillespie RJ, Knapik DM, Akkus O (2016) Biologic and synthetic grafts in the reconstruction of large to massive rotator cuff tears. J Am Acad Orthop Surg 24: 823-828.

7. Mihata T, McGarry MH, Pirolo JM, et al. (2012) Superior capsular reconstruction to restore superior stability in irreparable rotator cuff tears: A biomechanical cadaveric study. Am J Sports Med 40: 2248-2255.

8. Elhassan BT, Cox RM, Shukla DR, et al. (2017) Management of failed rotator cuff repair in young patients. J Am Acad Orthop Surg 25: 261-271.

9. Ellman H, Hanker G, Bayer M (1986) Repair of the rotator cuff. End-result study of factors influencing reconstruction. J Bone Joint Surg Am 68: 1136-1144.

10. Hartzler RU, Burkhart SS (2017) Superior capsular reconstruction. Orthopedics 40: 271-280.

11. Petri M, Greenspoon JA, Millett PJ (2015) Arthroscopic superior capsule reconstruction for irreparable rotator cuff tears. Arthrosc Tech 4: e751-e755.

12. S L (2015) Biomechanical testing of arthrex allograft vs. Fascia lata autograft as a suitable medium for superior capsular reconstruction. 16: 8 .

13. Radhakant Pandey, Suhayl Tafazal, Srinivasan Shyamsundar, et al. (2017) Outcome of partial repair of massive rotator cuff tears with and without human tissue allograft bridging repair. Shoulder Elbow 9: 23-30.

14. Mihata T, Lee TQ, Watanabe C, et al. (2013) Clinical results of arthroscopic superior capsule reconstruction for irreparable rotator cuff tears. Arthroscopy 29: 459-470.

15. John M Tokish, Clint Beicker (2015) Superior capsule reconstruction technique using an acellular dermal allograft. Arthrosc Tech 4: e833-e839.

16. Sethi P, Franco WG (2018) The role of superior capsule reconstruction in rotator cuff tears. Orthop Clin North Am 49: 93-101.

17. Hirahara AM, Andersen WJ, Panero AJ (2017) Superior capsular reconstruction: Clinical outcomes after minimum 2-year followup. Am J Orthop (Belle Mead NJ) 46: 266-278.

18. Mihata T, McGarry MH, Kahn T, et al. (2016) Biomechanical effect of thickness and tension of fascia lata graft on glenohumeral stability for superior capsule reconstruction in irreparable supraspinatus tears. Arthroscopy 32: 418-426.

19. Burkhart SS, Denard PJ, Adams CR, et al. (2016) Arthroscopic superior capsular reconstruction for massive irreparable rotator cuff repair. Arthrosc Tech 5: e1407-e1418.

20. Thorsness R, Romeo A (2016) Massive rotator cuff tears: Trends in surgical management. Orthopedics 39: 145-151.

21. Pogorzelski J, Muckenhirn KJ, Mitchell JJ, et al. (2017) Biomechanical comparison of 3 Glenoid-Side fixation techniques for superior capsular reconstruction. Am J Sports Med 46: 801-808.

22. Tokish JM, Momaya A, Roberson T (2018) Superior capsular reconstruction with a partial rotator cuff repair: A case report. JBJS Case Connect 8: e1. 
23. Dines JS (2017) Editorial commentary: A bridge to nowhere?...Do patches help improve our outcomes after rotator cuff surgery? Arthroscopy 33: 681-682.

24. Longo UG, Lamberti A, Maffulli N, et al. (2010) Tendon augmentation grafts: A systematic review. Br Med Bull 94: 165-188.

25. Jones CR, Snyder SJ (2015) Massive irreparable rotator cuff tears: A solution that bridges the gap. Sports Med Arthrosc Rev 23: 130-138.

26. Sutter EG, Godin JA, Garrigues GE (2017) All-arthroscopic superior shoulder capsule reconstruction with partial rotator cuff repair. Orthopedics 40: e735-e738.

27. McCormack RA, Shreve M, Strauss EJ (2014) Biologic augmentation in rotator cuff repair--should we do it, who should get it, and has it worked? Bull Hosp Jt Dis 72: 89-96.

28. Hirahara AM, Adams CR (2015) Arthroscopic superior capsular reconstruction for treatment of massive irreparable rotator cuff tears. Arthrosc Tech 4: e637-e641.

29. Narvani AA, Consigliere P, Polyzois I, et al. (2016) The "Pull-Over" technique for arthroscopic superior capsular reconstruction. Arthrosc Tech 5: e1441-e1447.

30. Denard PJ, Ladermann A, Jiwani AZ, et al. (2012) Functional outcome after arthroscopic repair of massive rotator cuff tears in individuals with pseudoparalysis. Arthroscopy 28: 1214-1219.

31. Saltzman BM, Chalmers PN, Gupta AK, et al. (2014) Complication rates comparing primary with revision reverse total shoulder arthroplasty. J Shoulder Elbow Surg 23: 1647-1654.

32. Farshad M, Gerber C (2010) Reverse total shoulder arthroplastyfrom the most to the least common complication. Int Orthop 34: 1075-1082.

33. Omid R, Lee B (2013) Tendon transfers for irreparable rotator cuff tears. J Am Acad Orthop Surg 21: 492-501. 